\title{
An unusual case of pain in left flank: renal hydatid cyst
}

\begin{abstract}
Echinococcal infection of kidney is very rare,1-3 more over involvement of the kidney without the involvement of the liver and lungs is even more rare. 2 Liver is the most common site of echinococcal infestation ( $54 \%$ to $77 \%$ ), because the liver acts as the initial filter for the organisms. Those larvae that escape the liver are next filtered by the lungs $(9 \%$ to $30 \%)$. The spleen, $(0.9 \%$ to $8 \%)$, kidney $(2 \%$ to $3 \%)$, and brain $(1 \%)$ are other organs involved.
\end{abstract}

Keywords: hydatid cyst kidney, echinococcus granulosus, scolicidal agent, pericystectomy, abdomen, ultra sonography
Volume 4 Issue | - 2017

\author{
Govind Yadav \\ Rd Gardi Medical College, India
}

Correspondence: Govind Yadav, Rd Gardi Medical College, Ujjain, Sector-40, Gurgaon, Haryana, India,

Email yadav.govind6@gmail.com

Received: October 30, 2016 | Published: February 28, 2017

\section{Case report}

A 15 year girl presented with complains of pain in left lumbar and left iliac fossa for 15days. Pain was sudden in onset and progressive, colicky in nature no aggravating or relieving factor. Not associated with nausea, vomiting or fever. There were no urinary complains. Ultra sonography (USG) abdomen showed a $9.1 \times 7.4 \times 7.2 \mathrm{~cm}$ well defined cystic lesion is seen in left hypochondrium abutting left kidney, spleen, tail of pancreas and stomach. A tiny medullary projection is seen from kidney into cyst? Renal cortical cyst/mesenteric cyst/pseudo cyst. USG followed by Computed tomography (CT) of abdomen which revealed large thin walled cystic lesion on left side of midline most likely arising from antero-superior aspect of left kidney. With these findings we made a diagnosis of simple cyst arising from left kidney. So surgery was planned.

\section{Surgical procedure}

Ureteric catheter was placed cystoscopically. Kidney was explored using left sub costal flank incision. A cyst was present on upper pole of kidney. The cyst content aspirated and was suggestive of hydatid cyst (CLEAR ASPIRATE). Whole cyst was irrigated with hypertonic saline de-roofing of cyst along with removal of endocyst and daughter cysts was done. After de-roofing of cyst betadine mixed saline was instilled through ureteric catheter and we recognized a rent in pelvicalyceal system which was repaired. Patient recovered completely and histopathology confirmed hydatid cyst. Patient was put on Albendazole 400mg twice daily for 3 cycles of 28days each cycle followed by a gap of 14 days.

\section{Differential diagnosis left flank pain}

Renal stones, parenchymal infection or inflammation, neuropathic pain, pleural pain, slipping rib syndrome, renal abscess, renal infarction.

\section{Discussion}

A typical hydatid cyst is composed of 3layers: a pericyst, an exocyst, and an endocyst (germinal layer) containing daughter cysts scolices and hooklets. Renal hydatid disease is an incidental finding, and patients often present with nonspecific clinical signs or symptoms. Presenting complaints are usually dull flank pain, hematuria, or palpable mass in flank region. Serology and imaging modalities establish the diagnosis in most of the cases. ${ }^{3,5}$ Serology consists of immuno-electrophoresis, immuno-hem agglutination test, and complement fixation test. The Casoni test is unreliable and outdated. A combination of investigations yields a diagnosis in only $50 \%$ of cases. Hydatid cyst can be evaluated by USG, IVU (intravenous urography), CT, or MRI(magnetic resonance image). ${ }^{3}$ USG and CT show a uni-loculated or a multi loculated cyst that may have some heterogenous echoes on USG or have rim enhancement on CT. ${ }^{3} \mathrm{~T} 1$ and T2-weighted MRI can be used to demonstrate the typical signs of hydatid disease. But in our case USG as well as CT did not provide any evidence of hydatid disease and no history of any animal contact was present. It was during operation only after aspiration we suspected and on deroofing we realized this is a case of hydatid cyst of kidney.

Percutaneous evacuation of a cyst is an option, but there is controversy concerning the management of the residual cavity following the procedure and risk of allergic reactions, including anaphylactic shock and dissemination. Scolicidal agent injection has been used successfully. Surgery is the treatment of choice in renal hydatid cyst. ${ }^{1,5}$ Kidney-conserving surgery like removal of hydatid cyst with pericystectomy is possible in most cases (75\%). Partial nephrectomy, nephrectomy must be reserved for destroyed kidney. ${ }^{5}$ Very few cases of laparoscopic removal of renal hydatid are reported. ${ }^{4}$ Utmost care should be taken during the surgery to prevent spillage and resultant disseminated hydatidosis. Pre and postoperative one-month courses of Albendazole should be considered in order to sterilize the cyst (decrease the chance of anaphylaxis) and decrease the tension in the cyst wall (thus reducing the risk of spillage during surgery) and to reduce the recurrence rate postoperatively. During kidney-sparing surgery scolicidal solutions such as hypertonic saline should be used before opening the cavities to kill the daughter cysts and therefore prevent further spread or anaphylactic reaction. 


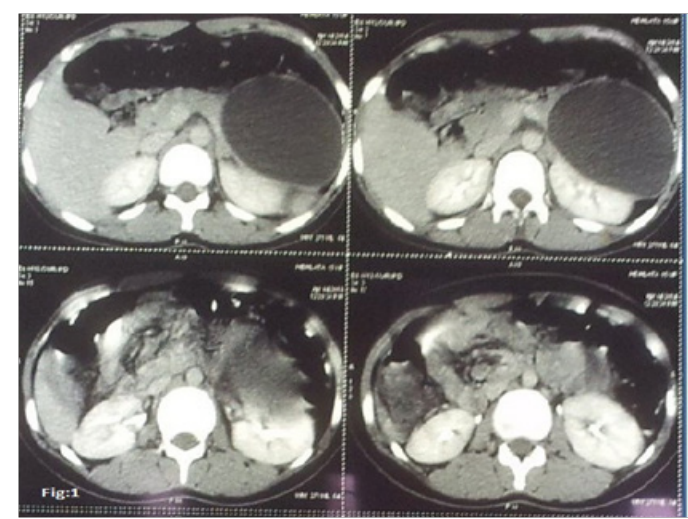

Figure I CT showing cyst at upper pole of left kidney.

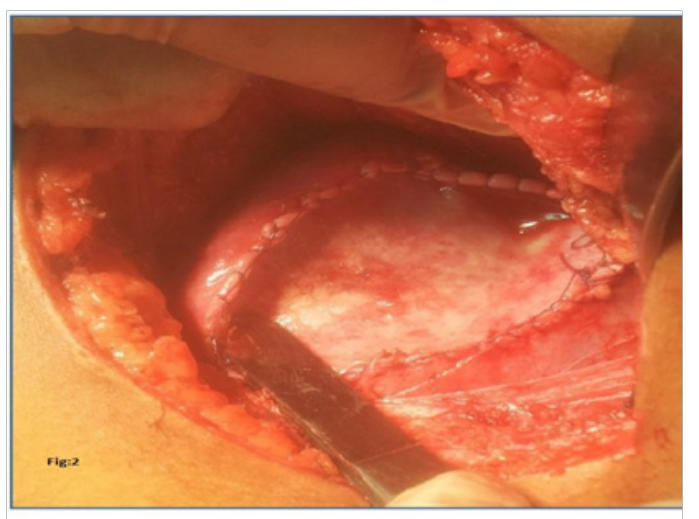

\section{Conclusion}

Keep high suspicion while dealing with renal cysts. Ureteric catheterization helps to identify ureter and any rent in pelvicalyceal system.

\section{Acknowledgements}

None.

\section{Conflict of interest}

The author declares no conflict of interest.

\section{References}

1. Volders WK, Gelin G, Stessens RC. Hydatid cyst of the kidney: Radiologicpathologic correlation. Radiographics. 2001;21(1):S255-260.

2. Unsal A, Cimentepe E, Dilmen G, et al. An unusual cause of renal colic: Hydatiduria. Int J Urol. 2001;8(6):319-321.

3. Sajid Ansari, Kanchan Dhungel, Kaleem Ahmad et al. Renal hydatid cyst: typical features on sonography. Journal of Pioneering Medical Sciences. 2013;3(4).

4. Karthik J Shah, Ganpule AP, Desai MR. Isolated renal hydatid cyst managed by laparoscopic transperitoneal nephrectomy. Indian $J$ urol. 2009;25(4):531-533

5. Pankaj Gharde, Wagh DD. Left renal Hydatid cyst presenting as haematuria and macroscopic hydatiduria since last ten years. Trop Parasitol. 2012;2(1):58-60.

6. Migliore M. Flank pain caused by slipping rib syndrome. Lancet. 2014;383(9919):844

Figure 2 Intraoperative cyst. 